\title{
Determinants of Coronary Vascular Events Responsible for Sudden Death- Vulnerable Plaque Trial (DISCOVER-VP)
}

\author{
Rachel Rivera \\ New Rochelle High School, 265 Clove Road, New Rochelle, New York, 10801, USA; rrivera10095@gmail.com
}

ABSTRACT: Annually, 500,000 deaths are attributed to cardiac arrests. Of these cases, $66 \%$ are caused by the accumulation of vulnerable plaque. This plaque increases the chance of a rupture exposing the necrotic core to blood flow and initiating a clot. ${ }^{1}$ Although vulnerable plaque poses a significant public health risk, its existence is usually unknown until death. The purpose of this study was to devise a method for vulnerable plaque identification among living patients using computed tomography (CT) scans of postmortem hearts and comparing single energy computed tomography (SECT) to dual energy computed tomography (DECT). The hearts underwent histopathology to determine the locations of all components. The arterial segments were sliced based on cross sections in regions of interest. These locations corresponded to specific areas on the CT scan, whose Hounsfield $(\mathrm{HU})$ units were subsequently compared. It was found that the mean $\mathrm{HU}$ of necrotic cores within vulnerable plaque of various regions of the ostium significantly differed from that of fibrous plaque on a DECT scan. ${ }^{2}$ This data suggests that DECT scans can be used to accurately distinguish dangerous vulnerable plaque from stable plaque in living patients when compared to SECT scans.

KEYWORDS: Cardiovascular Diseases; Sudden Cardiac Death; Coronary Computed Tomography; Unstable Plaque; Vulnerable Plaque.

\section{Introduction}

The American Heart Association approximates that 17.9 million people die of cardiovascular diseases annually and 500,000 of which are attributed to sudden cardiac death (SCD). ${ }^{3} \mathrm{SCD}$ is characterized by the fatal sudden loss of heart function. Several underlying factors, such as coronary artery disease and ventricular fibrillation, contribute to SCD. However, the primary cause of SCD, responsible for more than $66 \%$ of cases, is thrombosis, or the clotting, of vulnerable plaque. Vulnerable plaque is a type of atherosclerotic plaque developed within arterial segments in high-risk cardiovascular patients. ${ }^{4}$ The presence of vulnerable plaque is often detected only after death. As the cases of cardiac episodes, especially cardiac arrest, ${ }^{1}$ continue to increase exponentially, the urgency to take preventative action is critical.

Vulnerable plaque (VP) is identified by enlarged lipid necrotic core tissue, spotty calcification, and remodeling of the arteries due to plaque burden. ${ }^{5}$ VPs are also comprised of an enlarged lipid pool and thin fibrous cap (Figure 1). VP begins development as unstable VP. Unstable plaque is prone to rupturing and clotting. ${ }^{6}$ After rupturing, these plaques slowly gain stability and fully become calcified. ${ }^{6}$ In contrast, fibrous plaque, a common type of stable plaque, generally consists of a smaller necrotic core and thicker fibrous cap (Figure 1). The VP rupture exposes the necrotic core to blood flow, resulting in extensive clotting. ${ }^{7}$ The thin fibrous cap occasionally erodes, exposing the necrotic core to the inner lining of the arterial segment called the lumen. Macrophages attempt to restrict this exposure by forming a clot. The presence of VP is asymptomatic before the initial acute cardiac episode making it difficult to identify living patients with VP before the onset of SCD.$^{8}$ Previous research has identified that the lipid pool is a precursor for an unstable necrotic core. ${ }^{8}$ This region is highly unstable and exposed blood flow can result in fatal clotting. It is urgent for cardiologists to distinguish VP from stable plaque in living patients to ultimately prevent death.
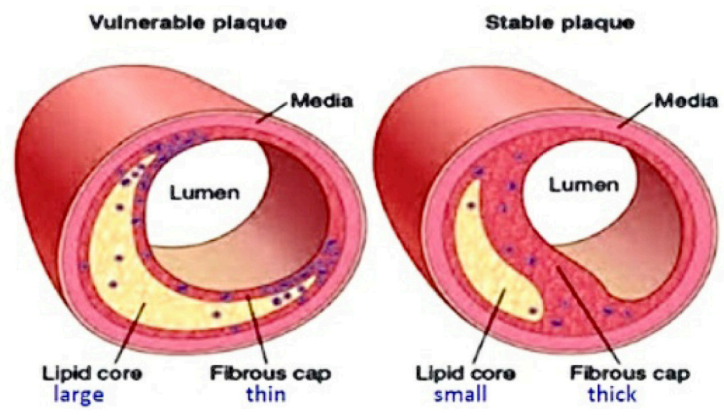

Figure 1: Vulnerable plaque with enlarged lipid core and thin fibrous cap (left) and stable plaque with thick fibrous cap and small lipid core (right). ${ }^{4}$

Coronary computed tomography angiography (CCTA) is commonly used for the characterization of atherosclerotic plaque, the determination of calcium scores, the precise identification of stenosis, and the diagnosis of plaques. Calcium scores refer to calcium accumulation within coronary segments. A high calcium score indicates the presence of extensive stable plaque and is a marker for coronary artery disease. Computed tomography (CT) scans can precisely identify stenosis and plaques. The two predominant $\mathrm{CT}$ scan types are single energy CT scans (SECT) and dual energy CT scans (DECT). Both scans are used to visualize coronary anatomy and eliminate calcium artifacts typical of other scans (Figure 2). 


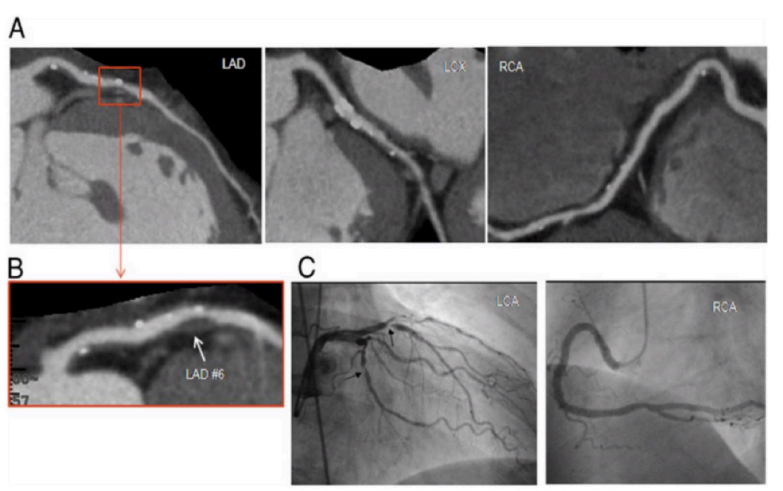

Figure 2: A) calcification of the left anterior descending artery (LAD), left circumflex, and right coronary artery; B) positive remodeling in the LAD; C) acute coronary syndrome 6 months after a CCTA. ${ }^{8}$

SECT scans are often used for diagnosis. Alternatively, DECT has the potential to more accurately identify various atherosclerotic plaques and other landmarks within the heart thanks to its higher resolution images. ${ }^{9,11}$ The current study compared the two scans to determine which one can best distinguish between VPs and stable plaques through the identification of high-risk plaque features. Since SECT is limited by its inability to effectively evaluate the characteristics of high-risk plaque, the DECT scan is preferable for VP identification and tissue characterization.

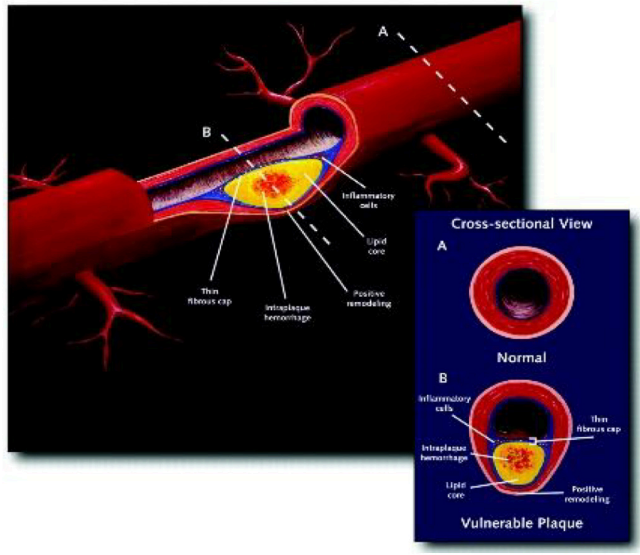

Figure 3: Composition of vulnerable plaque. ${ }^{5}$

Histopathology is another useful method for analyzing different plaque tissue characterization. This technique studies cross-sectionalized arterial segments under a microscope. ${ }^{12}$ Such visualization results in precise identification of each component, shown in Figure 3.

\section{Objective:}

To improve current methods of high-risk plaque diagnosis in SCD-suspected patients by comparing the accuracy of DECT and SECT during tissue characterization of VP.

\section{Hypothesis:}

H1. DECT will be more precise in identifying and characterizing VP ex-vivo in comparison to a SECT scan.

H2. DECT will more accurately characterize tissue of VP.

H3. High-risk plaque features will be identifiable from stable plaque features as expected to be justified through histology.

\section{Results and Discussion}

\section{DECT/SECT Identification of Necrotic Cores \& Fibrous}

\section{Plaques:}

Ninety-four cross sections were identified. The sample was made up of $71 \%$ male subjects and $29 \%$ female subjects. The mean age of the cohort was $61.4 \pm 10.9$ years. From this, a total of 8 necrotic cores (NC), 50 fibrous plaques (FP) or atherosclerotic plaques, and 36 lipid pools (LP) were identified using histopathology. Hounsfield units were computed by GE software to measure the substances' radiodensity. ${ }^{2}$ The mean HU for both the NC and FP using SECT was 29.4 and 78.95, respectively. The highest resolution to differentiate these two features was at $40 \mathrm{keV}$ of energy using a DECT scan. At 40 $\mathrm{keV}$, cardiologists are best able to differentiate between the stable and unstable VPs. On the other hand, the mean HU for the NC and FP for a DECT scan was 85.34 and 210.57, respectively, also at $40 \mathrm{keV}$. These values indicate that DECT scans are superior at $\mathrm{NC}$ and FP identification. A higher $\mathrm{HU}$ value coincides with a more precise identification of the examined substance on any CT scan. There was no notable difference for the mean values for LP.

Histopathology confirmed cardiologists' prediction that DECT scans are better at the prediction of vulnerable plaques within a heart. This correlates to observations on both the SECT and DECT scans since the position of the NCs, LP, and FPs aligned almost perfectly with the images produced following histopathology, illustrated in Figure 4.

With supporting results, it can be concluded that DECT scans more accurately identify VPs from stable plaques with the aid of high-risk plaque markers.

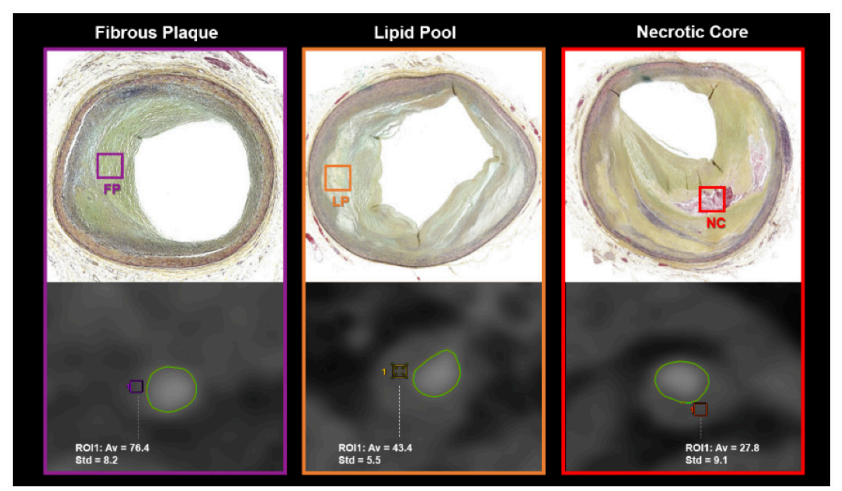

Figure 4: Accuracy of the predicated location of NC, LP, and FP on CT scans and histopathology images. ${ }^{2}$

\section{- Conclusion}

Based on the experiment's results, the hypothesis is supported. DECT scans are most effective at identifying VPs in the post-mortem hearts used in the study. Using histopathology, the presence of VPs was identified and mirrored the predictions of the plaque's lipid core and NC locations presented in the CT scans. Additionally, DECT scans were preferred because the resolution was better compared to SECT. Histopathology was useful to further examine the plaques and their 
compositions. It allowed for the measurements of NC, LP, and the thin fibrous cap. Furthermore, DECT using $40 \mathrm{keV}$ produced the most desirable results compared to a typical SECT scan.

DECT is the most efficient in distinguishing fibrous plaques from VPs through the identification of NC and LP location. This is groundbreaking for the cardiovascular medical field. Now that DECT has been shown to be most accurate, decreasing the high rates of mortality due to sudden cardiac death is almost at reach. DECT scans can be used to identify dangerous VPs within living patients who are susceptible to cardiovascular diseases. This allows doctors to take the necessary precautions to reduce a patient's risk of a potential cardiovascular disease thereby extending their life expectancy. Hundreds of thousands of lives can be saved through the simple identification of potentially hazardous plaques in highrisk cardiovascular patients.

\section{- Methods}

\section{Data Analysis \\ CT Scan Optimization:}

The goal of this step was to determine the reliability of CT scans using Youden's method. Youden's method allowed accuracy testing using the equation:

$\mathrm{J}=$ sensitivity + specificity -1

where $\mathrm{J}$ is the Youden test statistic. $\mathrm{J}=0$ indicates many false positive and false negative results and $\mathrm{J}=1$ indicates a perfect test with no errors. ${ }^{13}$ CT scans measure in Hounsfield units which measure a substance's radiodensity. DECT and SECT scans were each run at differing energy levels until $\mathrm{J}$ differed compared with paired t-tests. This test was performed with the assistance of the supervisor and J-values were unavailable to the student due to data privacy concerns. The statistical significance of the J-values indicated the reliability of both DECT and SECT scans for this experiment.

\section{Comparison of CT scan HU and Histopathology Values:}

Vessel and plaque parameters from histopathology were compared on SECT and DECT scans to a quantitative computed tomography. Histopathology analysis was computed using the Pearson correlation coefficient. The area of plaque components was measured directly with histopathology. This area was compared to the area of plaque components detected by optimally calibrated dual and single energy CT-scans with the Pearson correlation coefficient. A coefficient of 1 indicates a strong, positive relationship between the actual plaque component size and that measured while a coefficient of 0 indicates a weak relationship. The higher $r$-value indicated that the CT-scan accurately measured plaque components between the estimations and actual locations.

A two-sided $\mathrm{p}$-value was derived from each calculated correlation coefficient. This test was performed with the assistance of the supervisor and r-values were unavailable to the student due to data privacy concerns. This test indicated DECT scans accurately measure the size of vulnerable plaque components $(p<0.05)$. The correlation was calculated using the $95 \%$ confidence interval.

\section{All Other Variables:}

Continuous variables were represented with means or standard deviations, while categorical variables were expressed as percentages. Additionally, a separate equation was generated to account for the plaque features in all the individuals included within the study.

\section{Student and Mentor Roles :}

This study was a multi-site collaboration where each institute had separate responsibilities within the project. The earliest steps of the project were to go through the inclusion and exclusion criteria for each participant. Inclusion criteria consisted of newly deceased patients suspected of having SCD and meeting the minimum age requirement of 18 years. If eligible, the next of kin (NOK) provided recorded oral consent and requested an autopsy of the post-mortem heart.

My mentors oversaw each step within the study. After the heart was extracted, it underwent coronary cannulation where it was rinsed with normal saline to clean the heart. Contrast (Diatrizoate) was injected into the left and right main coronary artery at the Office of Chief Medical Examiner (OMCE) in Maryland. Following the coronary cannulation, the heart was taken to CVPath in Baltimore where SECT and DECT scans were performed. These images were taken seconds apart as the contrast flowed throughout the arteries within the heart. The images were sent to the Dailo Institute for Cardiovascular Imaging (DICI) where cardiologists used both the DECT and the SECT to determine total vessel area, NC area, calcified plaque area, luminal area, degree of positive remodeling, presence of spotty calcification with any mixed plaque, the presence of any napkin rings, intraluminal stenosis severity and proximal location by distance in millimeters to the coronary ostium. These values were obtained in HU. The cardiologists made annotations on these scans, directing incision points where a histopathologist performed histopathology during which the plaque was analyzed at a microscopic level. This allowed for better distinction between the stable and the vulnerable plaques identified within the arteries.

The student's role was to analyze the DECT and SECT scans of each ex-vivo heart and generate centerlines of each vessel (left anterior descending (LAD), left circumflex (LCX), right coronary artery (RCA), and the diagonals) using a reference CT scan for guidance. The student also observed any high-risk plaque features such as spotty calcification (Figure $3 \mathrm{~A}$ ) or positive remodeling (an extended diameter difference greater than $1 \mathrm{~mm}$ ) (Figure 3B). Additionally, the student assisted in the management of the data $\&$ measurements of the segments in HU. The mentor performed all other procedures.

\section{Acknowledgement}

Thank you to Dr. Fay Lin (principal investigator, Weill Cornell New York-Presbyterian, Dalio Institute of Cardiac Imaging) for allowing me to assist in your ongoing research. Thank you to Dr. Umberto Gianni (research fellow, Weill Cornell New York-Presbyterian, Dalio Institute of Cardiac Imaging) for deciding to have me on your team and having the patience to explain this study's complexity to me. I would also like to give a special thanks to June Thumpituk, Emma 
Hollenberg, Alexander Van Rosendael, Inge Van den Hoogen, Dr. Leslee Shaw, Dr. Benjamin Lee, Zahra Fatima, and Niree Hindoyan for your guidance and willingness to include me in additional projects. I would like to give a special thanks to my science research teacher, Mr. Wuebber. Thank you for your endless support and guidance through this journey!

\section{References}

1. Gillum, R.F. Sudden coronary death in the United States: 1980-1985. Circ. 1989, 79, 756-65. DOI: 10.1161/01.cir.79.4.756.

2. Amoa, F.; Dwivedi, A.: Al Hussein Alawaml, O.; Sato, Y.; Bax, A.; Elshafeey, A.; Gianni, U.; Wijeratne, R.; Hollenberg, E.; Lu, Y; et al. Lab Record. Weill Cornell NewYork-Presbyterian Dalio Institute of Cardiac Imaging, April 2018.

3. Chugh, S.S.; Reinier, K.; Teodorescu, C.; Evanado, A.; Kehr, E.; Al Samara, M.; Mariani, R., Gunson, K.; Jui, J. Epidemiology of Sudden Cardiac Death: Clinical and Research Implications. Prog. Cardiovasc. Dis. 2008, 51(3), 213-228. DOI: 10.1016/j.pcad.2008.06.003

4. Virmani, R.; Kolodgie, F.D.; Burke, A.P.; Farb, A; Scwartz, S.M. Lessons from sudden coronary death: a comprehensive morphological classification scheme for atherosclerotic lesions. Arterioscler. Thromb. Vasc. Biol. 2000, 20(5), 1262-1275. DOI: 10.1161/01.atv.20.5.1262

5. Bentzon, J.F; Otsuka, F.; Virmani, R.; Falk, E. Mechanisms of Plaque Formation and Rupture. Circ. Res. 2014, 114(12), 1852-1866. DOI: 10.1161/ CIRCRESAHA.114.302721.

6. Finn, A. V.; Nakano, M.; Narula, J., Kolodgie,; F. D.; Virmani, R, Concept of Vulnerable/Unstable Plaque. Arteriosclerosis, Thrombosis, and Vascular Biology 2010, 30(7), 1282-1292. DOI: 10.1161/atvbaha.108.179739.

7. Al'Aref, S.J.; Su, A.; Gransar, H.; Rosendael, A.R.; Rizvi, A.; Berman, D.S.; Callister, T.Q.; DeLago, A.; Hadamitzky, M.; Hausleiter, J.; Al-Mallah, M.H.; Budoff, M.J.; Kaufmann, P.A.; et al. A cross-sectional survey of coronary plaque composition in individuals on non-statin lipid lowering drug therapies and undergoing coronary computed tomography angiography. J. Cardiovasc. Comput. Tomogr. 2019, 13(2), 99-104. DOI: 10.1016/j.cct.2019.01.015.

8. Otsuka, F.; Yasuda, S.; Noguchi, T.; Ishibashi-Ueda, H. Pathology of coronary atherosclerosis and thrombosis. Cardiovascular Diagnosis and Therapy. 2016, 6(4), 396-408. DOI:10.21037/cdt.2016.06.01.

9. Motoyama, S.; Sarai, M.; Harigaya, H.; Anno, H.; Inoue, K.; Hara, T.; Naruse, H.; Ishii, J.; Hishida, H.; Wong, N.D.; et al. Computed tomographic angiography characteristics of atherosclerotic plaques subsequently resulting in acute coronary syndrome. J. Am. Coll. Cardiol. 2009, 54(1), 49-57. DOI: 10.1016/j.acc. 2009.02.068.

10. Nakanishi, R.; Baskaran, L.; Gransar, H.; Budoff, M.J.; Achenbach, S.; Al-, Mallah, M.; Cademartiri, F.; Callister, T.Q.; Chang H., Chinnaiyan, K.; et al. Relationship of Hypertension to Coronary. Atherosclerosis and Cardiac Events in Patients with Coronary Computed Tomographic Angiography. Hypertension 2017, 70(2), 293-299.

DOI: 10.1161/HYPERTENSIONAHA.117.09402 11. Bortnick, A.E.; Bartz, T.M.; Ix, J.H.; Chonchol, M.;
Reiner, A.; Cushman, M.; Owens, D.; Barasch, E.; Siscovick, D.S.; Gottdiener, J.; et al. Association of inflammatory, lipid and mineral markers with cardiac calcification in older adults. Heart 2016, 102(22), 1826-1834. DOI: 10.1136/heartjnl-2016-309404.

12. Narula, J.; Nakano, M.; Virmani, R; Kolodgie, F.D.; Petersen, R.; Newcomb, R.; Malik, S.; Fuster, V.; Finn, A.V. Histopathologic characteristics of atherosclerotic coronary disease and implications of the findings for the invasive and noninvasive detection of vulnerable plaques. J. Am. Coll. Cardiol. 2013, 61(10), 1041-1051. DOI: 10.1016/ j.jacc.2012.10.054.

13. Ruopp, M.D.; Perkins, N.J.; Whitcomb, B.W.; Schisterman, E.F. Youden Index and optimal cut-point estimated from observations affected by a lower limit of detection. Biom. J. 2008, 50(3), 419-430.

DOI: $10.1002 /$ bimj.200710415.

\section{- Authors}

Rachel Rivera is a junior from New Rochelle High School who anticipates following a pre-med track in college. Throughout her junior year, Rachel qualified for multiple regional competitions including selection as an international GENIUS Olympiad finalist. 